\title{
Real rectifiable currents, holomorphic chains and algebraic cycles
}

https://doi.org/10.1515/coma-2020-0119

Received December 24, 2020; accepted September 2, 2021

Abstract: We study some fundamental properties of real rectifiable currents and give a generalization of King's theorem to characterize currents defined by positive real holomorphic chains. Our main tool is Siu's semicontinuity theorem and our proof largely simplifies King's proof. A consequence of this result is a sufficient condition for the Hodge conjecture.

Keywords: real rectifiable current, real holomorphic chain, holomorphic subvariety, Hodge conjecture

MSC: 32U40, 14C25

\section{Introduction}

Since the publication of the foundational paper "Normal and integral currents" [7] by Federer and Fleming, geometric measure theory becomes an important tool in many areas of mathematics [5]. One particular fascinating question to us is to find characterization of currents defined by analytic varieties, or more generally, by holomorphic chains, which are some formal linear combination of analytic varieties. The first major progress was made by King in his marvelous paper [14] where he proved that holomorphic chains with positive integral coefficients are those $d$-closed rectifiable positive currents. Three years later Harvey and Shiffman improved King's result [13] showing that $d$-closed rectifiable currents of type $(k, k)$ with $(2 k+1)$-Hausdorff measure 0 support are integral holomorphic chains. They also conjectured that the condition on support is not necessary. This conjecture was affirmatively solved about twenty years later by Alexander [1]. So for holomorphic chains with integral coefficients, the characterization is complete. For holomorphic chains with real coefficients, as far as we know, not much is known. A significant difference lies in the fact that for an integral current $T$, its density $\Theta(\|T\|, x)$ is a nonnegative integer, but for a real rectifiable current $R$, its density $\Theta(\|R\|, x)$, is a nonnegative real number [6]. For example consider the real rectifiable current $\sum_{n=1}^{\infty} \frac{1}{2^{n}}\left[\frac{1}{n}\right]$ where $\left[\frac{1}{n}\right]$ is the current defined by the point $\frac{1}{n}$. This current is obviously a $d$-closed, positive, type $(0,0)$ real rectifiable current but it can not be a holomorphic 0-chain on $\mathbb{C}$ since the sequence $\left\{\frac{1}{n}\right\}_{n=1}^{\infty}$ has a limit point 0 and hence it can not be a holomorphic subvariety of $\mathbb{C}$. This means that the 3 conditions: $d$-closedness, real rectifiability and positivity are not sufficient to characterize positive real holomorphic chains. The main point of this paper is to show that in addition to the three conditions mentioned above, for a current $R$ to be a positive real holomorphic chain, we need an extra condition that the set of positive density $N:=\{x \mid \Theta(\|R\|, x)>0\}$ is $\mathcal{H}^{2 k}$ locally finite. Restriction on supports also appears naturally in studying plurisubharmonic positive currents $[3,11,12]$. We then show that currents with these four properties are positive real holomorphic chains. The following is our main result (Theorem 3.10).

\footnotetext{
*Corresponding Author: Jyh-Haur Teh: Department of Mathematics, Tsing Hua University(Hsinchu), 101 KuangFu Road, Section 2, 30013 Hsinchu, Taiwan, E-mail: jyhhaur@math.nthu.edu.tw

Chin-Jui Yang: Department of Mathematics, Tsing Hua University(Hsinchu), 101 KuangFu Road, Section 2, 30013 Hsinchu, Taiwan, E-mail: p9433256@gmail.com
} 
Theorem. Let $X$ be a complex manifold. If $T \in R R_{k, k}^{\text {loc }}(X)$ is positive, $d$-closed and $N=\left\{x \in X: \Theta^{2 k}(\|T\|, x)>\right.$ $0\}$ is $\mathcal{H}^{2 k}$-locally finite, then $T \in \mathscr{R}^{2} \mathscr{Z}_{k}^{+}(X)$.

The strategy to prove our main result is the following:

1. We first prove an integral representation theorem for real rectifiable currents;

2. we use this representation to show that $\operatorname{spt}(T)=\bar{N}$;

3. for $T$ positive and $d$-closed, we use Siu's semicontinuity theorem to decompose $N$ into a countable union $\bigcup_{n=1}^{\infty} E_{n}$ of holomorphic subvarieties, and apply the assumption that $N$ is $\mathcal{H}^{2 k}$-locally finite to show that $N$ is actually a holomorphic subvariety;

4. this implies that $N$ is a closed subset and hence $\operatorname{spt}(T)=N$ which proves our result.

Since the last condition in our main theorem that $N$ is $\mathcal{H}^{2 k}$-locally finite is automatically satisfied by positive integral currents, our result not only generalizes King's result but also our proof largely simplifies King's proof. This simplification is possible because of our use of Siu's famous semicontinuity theorem [19]. Techniques from geometric measure theory are already important tools in studying algebraic cycles [8, 9, $16,17]$. In our opinion, our characterization of real holomorphic chains may find important applications in studying the Hodge conjecture. We give a sufficient condition for homology classes that can be represented by algebraic cycles with rational coefficients on complex projective manifolds. In a forthcoming paper [21], we propose a version of the Hodge conjecture in Bott-Chern cohomology and use a generalized result of this paper to give a proof.

This paper is organized as follows. In section 2, we study some fundamental properties of real rectifiable currents. This includes an integral representation for real rectifiable currents which plays an important role in later development. We show that a locally normal current with $\mathcal{H}^{2 k}$-locally finite support is actually real rectifiable. In section 3, we give a generalization and at the same time, a new proof of King's theorem. In section 4, we apply our result to give a sufficient condition for homology classes to be represented by algebraic cycles with rational coefficients.

Acknowledgements. We thank the anonymous reviewer for his/her insightful comments and suggestions.

\section{Real rectifiable currents}

For $M$ a smooth oriented manifold, we denote by $A^{r}(M)$ the space of complex-valued smooth $r$-forms on $M$ and $A_{c}^{r}(M)$ the space of complex-valued $r$-forms with compact supports on $M$. Dually, $\mathscr{D}_{r}^{\prime}(M)$ is the space of currents of dimension $r$ and $\mathcal{E}_{r}^{\prime}(M)$ is the space of currents with compact supports.

Definition 2.1. Let $M$ be a smooth oriented manifold and $K \subset M$ be a compact set. A current $T \in \mathcal{E}_{r}^{\prime}(M)$ is called a real rectifiable r-current on $M$ with support in $K$ if for every $\varepsilon>0$, there is an open subset $U$ of some $\mathbb{R}^{n}$, a Lipschitz map $f: U \rightarrow M$ and a finite real polyhedral $r$-chain $P$ (in this article, we assume that simplices are nonoverlapping) with $f($ spt $P) \subset K$ such that the mass

$$
\mathbb{M}\left(T-f_{\star}(P)\right)<\epsilon
$$

Let $R R_{r, K}(M)$ be the space of real rectifiable $r$-currents on $M$ with supports in $K$ and the space of real rectifiable $r$-currents on $M$ is $R R_{r}(M)=\bigcup_{K} R R_{r, K}(M)$, where the union is taken over all compacta $K \subset M$. Locally real rectifiable r-currents on $M$ are elements of the set

$$
R R_{r}^{l o c}(M):=\left\{T \in \mathscr{D}_{r}^{\prime}(M): \text { for } x \in M \text {, there is } T_{x} \in R R_{r}(M) \text { such that } x \notin \operatorname{spt}\left(T-T_{x}\right)\right\} .
$$

We recall some definitions and results that we need later. Let $\mathcal{H}^{n}$ be the Hausdorff $n$-measure. 
Definition 2.2. Suppose that $N$ is a $\mathcal{H}^{n}$-measurable subset of $\mathbb{R}^{n+k}$ and $\theta$ is a positive locally $\mathcal{H}^{n}$-integrable function on $N$. We say that a given $n$-dimensional vector subspace $P$ of $\mathbb{R}^{n+k}$ is the approximate tangent space for $N$ at $x$ with respect to $\theta$ if for all $f \in A_{c}^{0}\left(\mathbb{R}^{n+k}\right)$,

$$
\lim _{\lambda \rightarrow 0} \lambda^{-n} \int_{N} f\left(\lambda^{-1}(z-x)\right) \theta(z) d \mathcal{H}^{n}(z)=\theta(x) \int_{P} f(y) d \mathcal{H}^{n}(y)
$$

The following result is [15, Proposition 5.4.3]. We recall that a set $M \subset \mathbb{R}^{n+N}$ is countably $n$-rectifiable if there exists $n$-dimensional embedded $C^{1}$ submanifolds $N_{1}, N_{2}, \ldots .$. and a set $N_{0} \subset \mathbb{R}^{n+N}$ with $\mathcal{H}^{n}\left(N_{0}\right)=0$ such that

$$
M \subset \bigcup_{k=0}^{\infty} N_{k}
$$

Proposition 2.3. Suppose that $M \subset \mathbb{R}^{n+k}$ is $\mathcal{H}^{n}$-measurable and countably n-rectifiable. Then $M=\coprod_{j=0}^{\infty} S_{j}$ where

1. $\mathcal{H}^{n}\left(S_{0}\right)=0$;

2. $S_{i} \cap S_{j}=\emptyset$ if $i \neq j$;

3. for $j \geq 1, S_{j} \subseteq N_{j}$ where $N_{j}$ is an $n$-dimensional, embedded $C^{1}$ submanifold of $\mathbb{R}^{n+k}$.

Recall that a $\mathcal{H}^{n}$-measurable function $\xi: M \rightarrow \Lambda_{n}\left(\mathbb{R}^{n+k}\right)$ is said to orient the approximate tangent space $T M$ of $M$ if for $\mathcal{H}^{n}$-almost everywhere $x \in M$, there exists an orthonormal basis $\left\{\tau_{1}, \ldots, \tau_{n}\right\}$ of $T_{x} M$ such that

$$
\xi(x)=\tau_{1} \wedge \cdots \wedge \tau_{n}
$$

The following result is [18, Theorem 11.6].

Theorem 2.4. Suppose that $N$ is $\mathcal{H}^{n}$-measurable. Then $N$ is countably $n$-rectifiable if and only if there is a positive locally $\mathcal{H}^{n}$-integrable function $\theta$ on $N$ with respect to which the approximate tangent space $T_{x} N$ exists for $\mathcal{H}^{n}$-almost every $x \in N$.

In the following, we generalize the integral representation theorem [7, Theorem 8.16] for integral currents to real rectifiable currents. This result plays a fundamental role in the later development.

Theorem 2.5. Let $T \in R R_{k}\left(\mathbb{R}^{n}\right)$. For all $\varphi \in A_{c}^{k}\left(\mathbb{R}^{n}\right)$,

$$
T(\varphi)=\int_{W}\langle\varphi(x), \vec{T}(x)\rangle \theta(x) d \mathcal{H}^{k}
$$

where $W$ is countably $k$-rectifiable and $\mathcal{H}^{k}$-measurable, $\theta: W \rightarrow \mathbb{R}$ is a positive $\mathcal{H}^{k}$-integrable function on $W,\|\vec{T}(x)\|=1$ and $\vec{T}(x)$ orients the approximate tangent space $T_{x} W$ for $\mathcal{H}^{k}$-almost every $x \in W$.

Proof. We follow the same strategy as Federer and Fleming proved [7, Theorem 8.16]. Let $C$ be the class of all $T \in \mathcal{E}_{k}^{\prime}\left(\mathbb{R}^{n}\right)$ which has the integral representation as stated. Let $f: \mathbb{R}^{k} \rightarrow \mathbb{R}^{n}$ be a function and $U \subset \mathbb{R}^{k}$ be an open convex set. The result will be proved in the following six steps:

1. If $f$ is continuously differentiable, $f$ is injective on the closure of $U$, and $(D f)_{u}$ is injective for $u \in U$, then $f_{\star}(U) \in C$.

2. If $T_{i} \in C$ for $i \in \mathbb{N}, \sum_{i=1}^{\infty} T_{i}=T \in \mathcal{E}_{k}^{\prime}\left(\mathbb{R}^{n}\right)$ and $\sum_{i=1}^{\infty} \mathbb{M}\left(T_{i}\right)<\infty$, then $T \in C$.

3. If $T_{i} \in C$ for $i \in \mathbb{N}$ and

$$
\lim _{i \rightarrow \infty} T_{i}=T \in \mathcal{E}_{k}^{\prime}\left(\mathbb{R}^{n}\right), \lim _{i \rightarrow \infty} \mathbb{M}\left(T_{i}-T\right)=0
$$

then $T \in C$.

4. If $f$ is continuously differentiable, then $f_{\star}(U) \in C$. 
5. If $f$ is Lipschitzian, then $f_{\star}(U) \in C$.

6. Every $k$-dimensional rectifiable current in $\mathbb{R}^{n}$ belongs to $C$.

Except 2, the other statements are proved similarly to [7, Theorem 8.16], so we only check the second statement in the following.

Let $\varphi \in A_{c}^{k}\left(\mathbb{R}^{n}\right)$. By the assumption

$$
T_{i}(\varphi)=\int_{W_{i}}\left\langle\varphi(x), \vec{T}_{i}(x)\right\rangle \theta_{i}(x) d \mathcal{H}^{k}, \quad \mathbb{M}\left(T_{i}\right)=\int_{W_{i}} \theta_{i}(x) d \mathcal{H}^{k}
$$

and

$$
T(\varphi)=\sum_{i=1}^{\infty} T_{i}(\varphi)=\sum_{i=1}^{\infty} \int_{W_{i}}\left\langle\varphi(x), \vec{T}_{i}(x)\right\rangle \theta_{i}(x) d \mathcal{H}^{k}
$$

Let $W=\bigcup_{i=1}^{\infty} W_{i}$ and extend each $\theta_{i}$ by zero outside $W_{i}$.

Since $\varphi$ has compact support, we may assume $\|\varphi\| \leq 1$. Note that $\left\|\vec{T}_{i}(x)\right\|=1$ for $\mathcal{H}^{k}$-almost every $x \in W_{i}$. So for any $n \in \mathbb{N}$,

$$
\sum_{i=1}^{n}\left|<\varphi(x), \vec{T}_{i}(x) \theta_{i}(x)>\right| \leq \sum_{i=1}^{\infty} \theta_{i}(x)
$$

By the Lebesgue dominated convergence theorem, we have

$$
\int_{W} \sum_{i=1}^{\infty} \theta_{i}(x) d \mathcal{H}^{k}=\sum_{i=1}^{\infty} \int_{W_{i}} \theta_{i}(x) d \mathcal{H}^{k}=\sum_{i=1}^{\infty} \mathbb{M}\left(T_{i}\right)<\infty
$$

which implies $\sum_{i=1}^{\infty} \theta_{i} \in L^{1}\left(\mathcal{H}^{k}\right)$.

By the Lebesgue dominated convergence theorem again,

$$
T(\varphi)=\int_{W}<\varphi(x), v(x)>d \mathcal{H}^{k}
$$

where $v(x)=\sum_{i=1}^{\infty} \theta_{i}(x) \vec{T}_{i}(x) \in \Lambda^{k}\left(T_{x} \mathbb{R}^{n}\right)$ is convergent.

By the hypothesis, for each $i \in \mathbb{N}$, there is a subset $Y_{i} \subset W_{i}$ such that for every $x \in Y_{i}, \vec{T}_{i}(x)$ exists, $\left\|\vec{T}_{i}(x)\right\|=1, \vec{T}_{i}(x)$ orients the approximate tangent space of $W_{i}$ at $x$, and $\mathcal{H}^{k}\left(W_{i} \backslash Y_{i}\right)=0$. Let $Y=\bigcup_{i=1}^{\infty} Y_{i}$. Since $W$ is countably $k$-rectifiable and $\mathcal{H}^{k}$-measurable, by Proposition 2.3, we may express $W$ as $W=\bigsqcup_{j=0}^{\infty} S_{j}$ where $S_{j} \subseteq N_{j}$ for some $C^{1}$-manifold $N_{j}$.

Let $Z=S_{0} \bigcup(W \backslash Y)$. Then $\mathcal{H}^{k}(Z)=0$. Fix $x \in W \backslash Z$. Then $x$ lies in some $N_{j}$. If $x \in W_{i} \cap W_{l}$, both $\vec{T}_{i}(x)$ and $\vec{T}_{\ell}(x)$ orient $T_{x} N_{j}$ which means $\vec{T}_{i}(x)= \pm \vec{T}_{\ell}(x)$. Since $\sum_{i=1}^{\infty} \theta_{i}(x) \vec{T}_{i}(x)$ converges in $\bigwedge^{k}\left(T_{x} N_{j}\right)$,

$$
\sum_{i=1}^{\infty} \theta_{i}(x) \vec{T}_{i}(x)=\sum_{i=1}^{\infty} \theta_{i}(x) a_{i}(x) \vec{T}_{\ell}(x)
$$

where $a_{i}(x)=1$ or -1 . Change all $a_{i}(x)$ to $-a_{i}(x)$ if necessary, we have

$$
\theta:=\sum_{i=1}^{\infty} a_{i} \theta_{i} \geq 0
$$

and $\sum_{i=1}^{\infty} \theta_{i}(x) \vec{T}_{i}(x)$ is of the form $\theta(x) \vec{T}(x)$ where $\vec{T}(x)= \pm \vec{T}_{\ell}(x)$ if $x \in N_{\ell}$. Since $\sum_{i=1}^{\infty} \theta_{i} \in L^{1}\left(\mathcal{H}^{k}\right)$ and all $\theta_{i}^{\prime} s$ are nonnegative, $\theta \in L^{1}\left(\mathcal{H}^{k}\right)$.

For all $\varphi \in A_{c}^{k}\left(\mathbb{R}^{n}\right)$,

$$
T(\varphi)=\int_{W}\langle\varphi(x), \vec{T}(x)\rangle \theta(x) d \mathcal{H}^{k}
$$

which means that $T \in C$. This completes the proof. 
The converse of the above result is also true.

Theorem 2.6. If $T \in \mathcal{E}_{k}^{\prime}\left(\mathbb{R}^{n}\right)$ and

$$
T(\varphi)=\int_{W}\langle\varphi(x), \vec{T}(x)\rangle \theta(x) d \mathcal{H}^{k}
$$

for all $\varphi \in A_{c}^{k}\left(\mathbb{R}^{n}\right)$ where $W$ is a countably $k$-rectifiable and $\mathcal{H}^{k}$-measurable set, $\theta$ is a positive $\mathcal{H}^{k}$-integrable function on $\mathbb{R}^{n},\|\vec{T}(x)\|=1$ and $\vec{T}(x)$ orients the approximate tangent space $T_{x} W$ for $\mathcal{H}^{k}$-almost every $x \in W$, then $T \in R R_{k}\left(\mathbb{R}^{n}\right)$.

Before we prove Theorem 2.6, we need a simple result whose validity is rather clear.

Lemma 2.7. Let $A$ be a bounded $\mathcal{L}^{n}$-measurable subset of $\mathbb{R}^{n}$. For any given $\varepsilon>0$, there is a finite set of disjoint $n$-simplices which coincide with A except for a set of measure less then $\varepsilon$.

Recall that if $U$ is an open subset of $\mathbb{R}^{k}$ and $f: U \rightarrow \mathbb{R}^{n}$ is a $C^{1}$ map, then $d^{U} f_{x}: T_{x} U \rightarrow \mathbb{R}^{n}$ is defined by

$$
d^{U} f_{x}(\tau):=\sum_{j=1}^{n}<\tau, \nabla^{U} f_{j}(x)>e_{j}
$$

where $f=\left(f_{1}, f_{2}, \ldots, f_{n}\right)$ and $\nabla^{U} f_{j}$ is the gradient of $f_{j}$ where $j=1,2, \ldots, n$ and $\left\{e_{1}, \ldots, e_{n}\right\}$ is the standard basis of $\mathbb{R}^{n}$.

Now we can prove Theorem 2.6.

Proof. Since $T$ has compact support, we may assume that $W$ is bounded. By Proposition 2.3, we may write $W=\coprod_{j=0}^{\infty} S_{j}$ where all $S_{j} \subseteq N_{j}$ have properties as stated in Proposition 2.3. We have

$$
\mathbb{M}(T)=\int_{W} \theta(x) d \mathcal{H}^{k}(x)=\sum_{i=1}^{\infty} \int_{S_{i}} \theta(x) d \mathcal{H}^{k}(x)=\sum_{i=1}^{\infty} \mathbb{M}\left(T\left\lfloor S_{i}\right)<\infty .\right.
$$

Given $\varepsilon>0$. Choose $m \in \mathbb{N}$ such that

$$
\sum_{i=m+1}^{\infty} \mathbb{M}\left(T\left[S_{i}\right)<\varepsilon\right.
$$

Fix $i$ with $1 \leq i \leq m$. Suppose that the $C^{1}$-manifold $N_{i}$ is parametrized by the $C^{1}$-diffeomorphism $f_{i}: U \rightarrow N_{i}$ for some open subset $U \subset \mathbb{R}^{k}$, and let $U$ be oriented by the natural orientation inherited from $\mathbb{R}^{k}$. For $x \in S_{i}$, there is $y \in U$ such that $f_{i}(y)=x$. Define

$$
\widetilde{\theta}_{i}(x)= \begin{cases}\theta(x), & \text { if } d^{U} f_{i, y^{\star}}\left(e_{1} \wedge \cdots \wedge e_{k}\right) \text { and } \vec{T}(x) \text { determine the same orientation. } \\ -\theta(x), & \text { otherwise. }\end{cases}
$$

Let

$$
\widetilde{T}_{i}(x)=\frac{d^{U} f_{i, y^{\star}}\left(e_{1} \wedge \cdots \wedge e_{k}\right)}{\left\|d^{U} f_{i, y^{\star}}\left(e_{1} \wedge \cdots \wedge e_{k}\right)\right\|}
$$

Then

$$
\left(T\left\lfloor S_{i}\right)(\varphi)=\int_{S_{i}}\left\langle\varphi(x), \widetilde{T}_{i}(x)\right\rangle \widetilde{\theta}_{i}(x) d \mathcal{H}^{k}(x)\right.
$$

Let $\widehat{\theta}_{i}=\widetilde{\theta}_{i} \circ f_{i}$. Then $\widehat{\theta}_{i}$ is Lebesgue integrable. By the change of variable formula, we have $T\left\lfloor S_{i}=f_{i^{\star}}\left(U \wedge \widehat{\theta}_{i}\right)\right.$. Given $\lambda_{i}>0$. Choose a simple function $\sum_{j=1}^{N} a_{j}^{i} \chi_{E_{j}^{i}}$ that is close to $\widehat{\theta}_{i}$ in $L^{1}$-norm where $a_{j}^{i} \in \mathbb{R}$ and all $E_{j}^{i} \subset \mathbb{R}^{n}$ are Lebesgue measurable such that

$$
\mathbb{M}\left(U \wedge \widehat{\theta}_{i}-U \wedge \sum_{j=1}^{N} a_{j}^{i} \chi_{E_{j}^{i}}\right) \leq\left\|\widehat{\theta}_{i}-\sum_{j=1}^{N} a_{j}^{i} \chi_{E_{j}^{i}}\right\|_{L^{1}(U)}<\lambda_{i}
$$


For each $j \in\{1, \ldots, N\}$, by Lemma 2.7, we can find finitely many disjoint polyhedrals $\Delta_{j, l}^{i}$ in $\mathbb{R}^{n}$ for $l=$ $1, \ldots, q_{j}$ such that

$$
\mathcal{H}^{k}\left(\left(E_{j}^{i} \backslash \bigsqcup_{l=1}^{q_{j}} \Delta_{j, l}^{i}\right) \bigcup\left(\bigsqcup_{l=1}^{q_{j}} \Delta_{j, l}^{i} \backslash E_{j}^{i}\right)\right)<\frac{\lambda_{i}}{N\left|a_{j}^{i}\right|}
$$

Let $P_{i}=\sum_{j=1}^{N} \sum_{l=1}^{q_{j}} a_{j}^{i} \Delta_{j, l}^{i}$. Then

$$
\mathbb{M}\left(U \wedge \sum_{j=1}^{N} a_{j}^{i} \chi_{E_{j}^{i}}-P_{i}\right) \leq \sum_{j=1}^{N} \int_{\left(E_{j}^{i} \backslash \bigsqcup_{l=1}^{q_{j}} \Delta_{l}^{j}\right) \cup\left(\bigsqcup_{l=1}^{q_{j}} \Delta_{l}^{j} \backslash E_{j}^{i}\right)}\left|a_{j}^{i}\right| d \mathcal{H}^{k}(x)<\lambda_{i}
$$

This implies that

$$
\mathbb{M}\left(T\left[S_{i}-f_{i_{*}} P_{i}\right) \leq \operatorname{Lip}\left(f_{i}\right)^{k}\left[\mathbb{M}\left(U \wedge \hat{\theta}_{i}-U \wedge \sum_{j=1}^{N} a_{j}^{i} \chi_{E_{j}^{i}}\right)+\mathbb{M}\left(U \wedge \sum_{j=1}^{N} a_{j}^{i} \chi_{E_{j}^{i}}-P_{i}\right)\right]<2 L i p\left(f_{i}\right)^{k} \lambda_{i} .\right.
$$

Now take $C=m\left(\max _{i=1, \ldots, m}\left\{\operatorname{Lip}\left(f_{i}\right)^{k}\right\}\right)$ and $\lambda_{i}=\frac{\varepsilon}{2 C}$. We have

$$
\mathbb{M}\left(T-\sum_{i=1}^{m} f_{i_{\star}} P_{i}\right) \leq \mathbb{M}\left(\sum_{i=m+1}^{\infty} T\left\lfloor S_{i}\right)+\sum_{i=1}^{m} \mathbb{M}\left(T\left\lfloor S_{i}-f_{i_{\star}} P_{i}\right)<2 \varepsilon\right.\right.
$$

This completes the proof.

Definition 2.8. 1. A triple $(W, \theta, \vec{T})$ is called an oriented real $k$-rectifold if $W$ is a countably $k$-rectifiable and $\mathcal{H}^{k}$-measurable set, $\theta$ is a positive locally $\mathcal{H}^{k}$-integrable function on $W, \vec{T}(x)$ orients the approximate tangent space $T_{x} W$ and $\|\vec{T}(x)\|=1$ for $\mathcal{H}^{k}$-almost every $x \in W$.

2. The real rectifiable current associated to an oriented real k-rectifold $(W, \theta, \vec{T})$ is the current $T \in R R_{k}^{\text {loc }}\left(\mathbb{R}^{n}\right)$ defined by

$$
T(\varphi)=\int_{W}\langle\varphi(x), \vec{T}(x)\rangle \theta(x) d \mathcal{H}^{k}
$$

for $\varphi \in A_{c}^{k}\left(\mathbb{R}^{n}\right)$.

We note that by Theorem 2.5, a real rectifiable $k$-current is naturally associated with an oriented real $k$ rectifold.

Definition 2.9. Let $U \subset \mathbb{R}^{n}$ be an open set. We say that a subset $A \subset U$ is $\mathcal{H}^{k}$-locally finite if for any $u \in U$, there is $r>0$ such that $\mathcal{H}^{k}\left(A \cap B_{r}(u)\right)<\infty$ where $B_{r}(u)$ is the open ball centered at $u$ with radius $r$.

We denote by $\Omega(m)$ the volume of the $m$-dimensional unit closed ball.

Definition 2.10. Let $\mu$ be a measure on $\mathbb{R}^{n}$. The m-dimensional upper density of $\mu$ at $p$ is

$$
\Theta^{\star m}(\mu, p):=\limsup _{r \rightarrow 0} \frac{\mu\left[\overline{B_{r}(p)}\right]}{\Omega(m) r^{m}}
$$

and the m-dimensional lower density of $\mu$ at $p$ is

$$
\Theta_{\star}^{m}(\mu, p):=\liminf _{r \rightarrow 0} \frac{\mu\left[\overline{B_{r}(p)}\right]}{\Omega(m) r^{m}}
$$

If $\Theta^{\star m}(\mu, p)=\Theta_{\star}^{m}(\mu, p)$, then we call their common value the $m$-dimensional density of $\mu$ at $p$ and denote it by $\Theta^{m}(\mu, p)$.

For a current $T$, we denote by $\|T\|$ the total variation of $T$. 
Proposition 2.11. Suppose that $T \in R R_{k}^{\text {loc }}\left(\mathbb{R}^{n}\right)$ is the real rectifiable $k$-current associated to an oriented real $k$-rectifold $(W, \theta, \vec{T})$. Let $N=\left\{x \in \mathbb{R}^{n}: \Theta^{k}(\|T\|, x)>0\right\}$. Then

$$
T(\varphi)=\int_{N}\langle\varphi(x), \vec{T}(x)\rangle \Theta^{k}(\|T\|, x) d \mathcal{H}^{k}
$$

for all $\varphi \in A_{c}^{k}\left(\mathbb{R}^{n}\right)$, and $W$ is $\mathcal{H}^{k}$-locally finite if and only if $N$ is $\mathcal{H}^{k}$-locally finite.

Proof. Let $\mu:=\mathcal{H}^{k}\lfloor\theta=\|T\|$. The existence of the approximate tangent plane (Theorem 2.4) of $W$ implies that (see [18, pg 63])

$$
0<\theta(x)=\lim _{r \rightarrow 0^{+}} \frac{\int_{W \cap B_{r}(x)} \theta(y) d \mathcal{H}^{k}(y)}{\Omega(k) r^{k}}=\Theta^{k}(\mu, x)
$$

for $\mu$-almost every $x \in W$. This implies that $\mathcal{H}^{k}(W-N)=0$.

Let

$$
\bar{\theta}(x)= \begin{cases}\theta(x), & \text { if } x \in W \\ 1, & \text { otherwise. }\end{cases}
$$

and $\bar{\mu}=\mathcal{H}^{k}\lfloor\bar{\theta}$.

Since $\theta$ is locally $\mathcal{H}^{k}$-integrable, for each $x \in W$, we have

$$
\mathbb{M}\left(T\left\lfloor B_{1}(x)\right)=\int_{W \cap B_{1}(x)} \theta d \mathcal{H}^{k}<\infty .\right.
$$

Since $\bar{\mu}$ is Borel regular, $W \subset \mathbb{R}^{n}$ is $\bar{\mu}$-measurable and

$$
\bar{\mu}\left(W \cap B_{1}(x)\right)=\int_{W \cap B_{1}(x)} \bar{\theta}(x) d \mathcal{H}^{k}(x)=\int_{W \cap B_{1}(x)} \theta(x) d \mathcal{H}^{k}(x)=\mathbb{M}\left(T\left\lfloor B_{1}(x)\right)<\infty,\right.
$$

by [18, Theorem 3.5],

$$
\Theta^{\star k}(\bar{\mu}, W, y)=\Theta^{\star k}\left(\bar{\mu}, W \cap B_{1}(x), y\right)=0
$$

for $\bar{\mu}$-almost every $y \in B_{1}(x)-W$. Find a sequence $\left\{x_{j}\right\}_{j=1}^{\infty}$ in $W$ such that $W \subset V=\bigcup_{j=1}^{\infty} B_{1}\left(x_{j}\right)$. Then

$$
\Theta^{\star k}(\bar{\mu}, W, y)=0
$$

for $\bar{\mu}$-almost every $y \in V-W$. Clearly, $\Theta^{\star k}(\bar{\mu}, W, y)=0$ for all $y \in \mathbb{R}^{n}-V$. Therefore $\Theta^{k}(\|T\|, x)=0$ for $\mathcal{H}^{k}$-almost every $x \in \mathbb{R}^{n}-W$. This implies $\mathcal{H}^{k}(N-W)=0$.

From the equality

$$
\mathcal{H}^{k}[(W \backslash N) \bigcup(N \backslash W)]=0
$$

we may rewrite

$$
T(\varphi)=\int_{W}<\varphi(x), \vec{T}(x)>d \mathcal{H}^{k}\left\lfloor\theta=\int_{N}<\varphi(x), \vec{T}(x)>\Theta^{k}(\|T\|, x) d \mathcal{H}^{k}\right.
$$

for all $\varphi \in A_{c}^{k}\left(\mathbb{R}^{n}\right)$ and we see that $W$ is $\mathcal{H}^{k}$-locally finite if and only if $N$ is $\mathcal{H}^{k}$-locally finite.

Theorem 2.12. If $T \in N_{k}^{l o c}\left(\mathbb{R}^{n}\right)$ has $\mathcal{H}^{k}$-locally finite support, then $T$ is real rectifiable.

Proof. Let $S=\operatorname{spt}(T)$. Since $S$ is $\mathcal{H}^{k}$-locally finite, by [7, pg 494 (4)],

$$
\Theta^{k}\left(\mathcal{H}^{k}, S, x\right)=\lim _{r \rightarrow 0^{+}} \frac{\mathcal{H}^{k}\left(S \bigcap B_{r}(x)\right)}{\Omega(k) r^{k}} \geq 2^{-k}
$$


for $\mathcal{H}^{k}$-almost every $x \in S$. By [18, Page 63]

$$
\begin{aligned}
\theta(x) & =\lim _{r \rightarrow 0^{+}} \frac{\|T\|\left(B_{r}(x)\right)}{\mathcal{H}^{k}\left(S \bigcap B_{r}(x)\right)}=\lim _{r \rightarrow 0^{+}} \frac{\|T\|\left(B_{r}(x)\right)}{\Omega(k) r^{k}} \frac{\Omega(k) r^{k}}{\mathcal{H}^{k}\left(S \bigcap B_{r}(x)\right)} \\
& \leq 2^{k} \lim _{r \rightarrow 0^{+}} \frac{\|T\|\left(B_{r}(x)\right)}{\Omega(k) r^{k}}=2^{k} \Theta^{k}(\|T\|, x)
\end{aligned}
$$

for $\|T\|$-almost every $x \in S$. Thus $\Theta^{k}(\|T\|, x)>0$ for $\|T\|$-almost every $x \in S$. Since $\|T\|\left(\mathbb{R}^{n}-S\right)=0$, $\Theta^{k}(\|T\|, x)>0$ for $\|T\|$-almost every $x \in \mathbb{R}^{n}$. By [18, Theorem 32.1], $T$ is real rectifiable.

\section{A generalization of King's theorem}

Definition 3.1. Suppose that $U \subset \mathbb{C}^{n}$ is an open subset. Let $\omega$ be the standard Kähler form of $\mathbb{C}^{n}, \omega_{k}=\frac{\omega^{k}}{k !}$ and $T \in \mathscr{D}_{k, k}^{\prime}(U)$ be a positive closed current. The Lelong number $n(T, a)$ of $T$ at a point $a \in U$ is defined to be $\Theta^{2 k}\left(T \wedge \omega_{k}, a\right)$.

Since we have integral representation for locally real rectifiable currents. The following result is a simple modification of [13, Lemma 1.12].

Lemma 3.2. Let $U \subset \mathbb{C}^{n}$ be an open subset. Suppose that $T \in R R_{2 k}^{\text {loc }}(U)$ is associated to the oriented real $2 k$-rectifold $(W, \theta(x), \vec{T}(x))$. Then $T \in R R_{k, k}^{\text {loc }}(U)$ if and only if $\vec{T}(x)$ is complex (i.e. $\vec{T}(x)$ represents a complex subspace of $\left.\mathbb{C}^{n}\right)$ for $\|T\|$-almost every $x \in U$. Furthermore, $T \in R R_{k, k}^{l o c}(U)$ is positive if and only if $\vec{T}(x)$ is complex and positive for $\|T\|$-almost every $x \in U$.

Lemma 3.3. Suppose that $U \subset \mathbb{C}^{n}$ is an open subset. If $T \in R R_{k, k}^{\text {loc }}(U)$ is positive and closed, then

$$
n(T, a)=\Theta^{2 k}(\|T\|, a)
$$

for all $a \in U$. In particular, $\Theta^{2 k}(\|T\|, a)$ exists for all $a \in U$.

Proof. By the integral representation (Theorem 2.5), for any Borel set $B \subset U$,

$$
(T \cap B)\left(\omega_{k}\right)=\int_{B}<\omega_{k}, \vec{T}>\theta d \mathcal{H}^{2 k}
$$

By Lemma 3.2, $\vec{T}(x)$ is complex and by Wirtinger's inequality [4, Theorem 4.1], $\left\langle\omega_{k}, \vec{T}\right\rangle=1$. Therefore

$$
(T \cap B)\left(\omega_{k}\right)=\int_{B} \theta d \mathcal{H}^{2 k}=\|T\|(B)
$$

In particular,

$$
\|T\|\left(B_{r}(a)\right)=\left(T \cap B_{r}(a)\right)\left(\omega_{k}\right)=\left(T \wedge \omega_{k}\right)\left(B_{r}(a)\right)
$$

Hence $\|T\|=T \wedge \omega_{k}$, and therefore $n(T, a)=\Theta^{2 k}(\|T\|, a)$. Since the Lelong number $n(T, a)$ exists for all $a \in U$, so does $\Theta^{2 k}\left(T \wedge \omega_{k}, a\right)$.

We recall that a closed subset $A \subset X$ in a complex manifold $X$ is a holomorphic subvariety if for any point $a \in A$, there is a neighborhood $W \subset X$ of $a$ and some $f_{1}, \ldots, f_{m} \in \mathcal{O}(W)$ such that

$$
A \cap W=\left\{x \in X: f_{1}(x)=\cdots=f_{m}(x)=0\right\}
$$

Definition 3.4. Let $X$ be a complex manifold. A current $T \in \mathscr{D}_{2 k}^{\prime}(X)$ is said to be a real holomorphic $k$-chain on $X$ if $T$ can be written in the form $T=\sum_{j=1}^{\infty} r_{j}\left[V_{j}\right]$ where $r_{j} \in \mathbb{R}$ and $V=\bigcup_{j=1}^{\infty} V_{j}$ is a purely $k$-dimensional holomorphic subvariety of $X$ with irreducible components $\left\{V_{j}\right\}_{j=1}^{\infty}$. The vector space of real holomorphic $k$-chains on $X$ is denoted by $\mathscr{R} \mathscr{Z}{ }_{k}(X)$. Denote by $\mathscr{R} \mathscr{Z}_{k}^{+}(X)$ the set of positive real holomorphic $k$-chains on $X$, i.e., those real holomorphic $k$-chains with nonnegative coefficients. 
We recall the following semicontinuity theorem of Siu's (see $[2,19])$.

Theorem 3.5. (Siu's semicontinuity theorem) If $T$ is a closed positive current of bidimension $(k, k)$ on a complex manifold $X$, then the upperlevel sets

$$
E_{c}(T)=\{x \in X: n(T, x) \geq c\}
$$

are holomorphic subvarieties of dimension $\leq k$.

For the convenience of the reader, we cite [20, Theorem B] that will be used in the proof of our next result.

Theorem 3.6. ([20, Theorem B]) If a purely $k$-dimensional subvariety of an $r$-ball in $\mathbb{C}^{n}$ passes through the center of the ball, then its $2 k$-volume is at least $\Omega(2 k) r^{2 k}$.

Proposition 3.7. Let $U$ be an open subset of $\mathbb{C}^{n}$ and $A_{i}$ be an irreducible holomorphic subvariety of dimension $k$ in $U$ for $i=1,2, \ldots$. If $A=\bigcup_{i=1}^{\infty} A_{i}$ is $\mathcal{H}^{2 k}$-locally finite, then $A$ is a holomorphic subvariety of $U$.

Proof. Suppose that there is a point $a \in U$ such that each neighborhood of $a$ meets infinitely many $A_{i}^{\prime}$ s. Fix any $r>0$ with $B_{2 r}(a) \subset U$. Then $B_{r}(a)$ meets infinitely many $A_{i}^{\prime}$ s. Assume that $B_{r}(a)$ meets $A_{i_{j}}, j=1,2, \ldots$ For each $\mathrm{j}$, there is a point $a_{j} \in A_{i_{j}}$ such that $\left\|a-a_{j}\right\|<r$. Hence $B_{r}\left(a_{j}\right) \subset B_{2 r}(a)$. By Theorem 3.6,

$$
\mathcal{H}^{2 k}\left(B_{2 r}(a) \cap A_{i_{j}}\right) \geq \mathcal{H}^{2 k}\left(B_{r}\left(a_{j}\right) \cap A_{i_{j}}\right) \geq \Omega(2 k) r^{2 k}
$$

Since

$$
\mathcal{H}^{2 k}\left(B_{2 r}(a) \cap A\right)=\lim _{m \rightarrow \infty} \mathcal{H}^{2 k}\left(B_{2 r}(a) \cap\left(\cup_{j=1}^{m} A_{i_{j}}\right)\right)
$$

and $A_{i_{j}} \cap A_{i_{k}}$ is of $\mathcal{H}^{2 k}$ measure 0 for $j \neq k$, by the inclusion-exclusion principle, we have

$$
\begin{aligned}
\mathcal{H}^{2 k}\left(B_{2 r}(a) \cap\left(\cup_{j=1}^{m} A_{i_{j}}\right)\right) & =\mathcal{H}^{2 k}\left(\cup_{j=1}^{m}\left(B_{2 r}(a) \cap A_{i_{j}}\right)\right)=\sum_{j=1}^{m} \mathcal{H}^{2 k}\left(B_{2 r}(a) \cap A_{i_{j}}\right) \\
& \geq \sum_{j=1}^{m} \Omega(2 k) r^{2 k}=m \Omega(2 k) r^{2 k}
\end{aligned}
$$

which approaches $\infty$ as $m \rightarrow \infty$. This is true for all $r$ sufficiently small, therefore $A$ is not $\mathcal{H}^{2 k}$-locally finite which is a contradiction.

Proposition 3.8. Let $X$ be a complex manifold. If $T \in R R_{k}^{l o c}(X)$ and $N=\left\{x \in X: \Theta^{k}(\|T\|, x)>0\right\}$, then $\operatorname{spt}(T)=\bar{N}$.

Proof. Since the result is local, we may assume $T \in R R_{k}(U)$ for some open subset $U \subset \mathbb{C}^{n}$. By Theorem 2.5 and Proposition 2.11,

$$
T(\varphi)=\int_{N}\langle\varphi(x), \vec{T}(x)\rangle \Theta^{k}(\|T\|, x) d \mathcal{H}^{k}
$$

for all $\varphi \in A_{c}^{k}(U)$. If $a \notin \bar{N}$, there is a neighborhood $V$ of $a$ such that $V \cap \bar{N}=\emptyset$. Hence for any $w \in A_{c}^{k}(U)$ with $\operatorname{spt}(w) \subset V$, we have $T(w)=0$. Therefore $V \subset(\operatorname{spt}(T))^{c}$, and this shows that $\bar{N}^{c} \subset(\operatorname{spt}(T))^{c}$, equivalently, $\operatorname{spt}(T) \subset \bar{N}$. On the other hand, for $a \in N, \Theta^{k}(\|T\|, a)>0$ implies that there are infinitely many $r>0$ such that

$$
\frac{\|T\|\left(B_{r}(a)\right)}{\Omega(k) r^{k}}>0
$$

For each such $r>0$, since

$$
\|T\|\left(B_{r}(a)\right)=\sup \left\{T(w): w \in A_{c}^{k}(U) \text { with }\|w\| \leq 1 \text { and } \operatorname{spt}(w) \subset B_{r}(a)\right\}
$$

we can find at least one $w \in A_{c}^{k}(U)$ with $\operatorname{spt}(w) \subset B_{r}(a)$ such that $T(w)>0$. This shows that $a \in \operatorname{spt}(T)$, and hence $\bar{N} \subset \operatorname{spt}(T)$ since $\operatorname{spt}(T)$ is closed in $U$. 
We need the following result from [14, Proposition 3.1.3].

Proposition 3.9. If $V$ is a $k$-dimensional holomorphic subvariety of a complex manifold $X$, then for any closed flat chain $T \in F_{2 k}^{\text {loc }}(X)$ with $\operatorname{spt}(T) \subset V, T$ is of the form $\sum_{j=1}^{\infty} a_{j}\left[V_{j}\right]$ where each $V_{j}$ is a global irreducible component of $V=\bigcup_{j=1}^{\infty} V_{j}$ and $a_{j} \in \mathbb{C}$.

Now we give a generalization of King's theorem to real rectifiable currents.

Theorem 3.10. Let $X$ be a complex manifold. If $T \in R R_{k, k}^{\text {loc }}(X)$ is positive, $d$-closed and $N=\{x \in X$ : $\left.\Theta^{2 k}(\|T\|, x)>0\right\}$ is $\mathcal{H}^{2 k}$-locally finite, then $T \in \mathscr{R} \mathscr{Z}_{k}^{+}(X)$.

Proof. The result is local, so it is enough to consider $X=U$ for some open subset $U \subset \mathbb{C}^{n}$. By Lemma 3.3, $N=\{x \in U: n(T, x)>0\}$. Write

$$
N=\bigcup_{n=1}^{\infty} E_{n} \text { where } E_{n}=\left\{x \in U: n(T, x) \geq \frac{1}{n}\right\}
$$

Then by Siu's semicontinuity theorem, $E_{n}$ is a holomorphic subvariety of $U$ with dimension $\leq k$ for all $n \in \mathbb{N}$. By the Measure Support Theorem ([14, Theorem 2.4.2]), we may assume that each $E_{n}$ is of purely $k$-dimensional. By the assumption, $N$ is of $\mathcal{H}^{2 k}$-locally finite and hence by Proposition 3.7, $N$ is a holomorphic subvariety and hence closed in $U$. By Proposition 3.8, $\operatorname{spt}(T)=\bar{N}=N$. Since $R R_{k}^{l o c}(U) \subset F_{k}^{l o c}(U)$, the result follows from Proposition 3.9.

In the following, we show that King's theorem is a simple consequence of our result.

Corollary 3.11. Let $X$ be a complex manifold.

1. Suppose that $T \in R R_{k, k}^{\text {loc }}(X)$ is positive, $d$-closed and

$$
N=\left\{x \in U: \Theta^{2 k}(\|T\|, x)>0\right\}
$$

is $\mathcal{H}^{2 k}$-locally finite. If $n(T, x) \in \mathbb{Z}$ (respectively $\left.\mathbb{Q}\right)$ for all $x \in X$, then $T$ is a holomorphic chain with positive integral (respectively rational) coefficients.

2. (King's theorem) Suppose that $T \in R_{k, k}^{l o c}(X)$ is positive and d-closed, then $T$ is a holomorphic chains with integral coefficients.

Proof. By Theorem 3.10, $T=\sum_{j=1}^{m} a_{j}\left[V_{j}\right]$ is a holomorphic chain for some positive real numbers $a_{j}^{\prime} s$ and irreducible $k$-dimensional holomorphic subvarieties $V_{j}^{\prime} s$ of $U$. For each $j$, choose $x_{j} \in V_{j}-\cap_{i \neq j} V_{i}$. Then $n\left(T, x_{j}\right)=a_{j} n\left(\left[V_{j}\right], x_{j}\right)$ and by Thie's theorem ([14, Theorem 4.2.2]), $n\left(\left[V_{j}\right], x_{j}\right)$ is a positive integer. This implies that if $n\left(T, x_{j}\right) \in \mathbb{Z}$ ( respectively $\left.\mathbb{Q}\right)$, then $a_{j} \in \mathbb{Z}($ respectively $\mathbb{Q})$.

2. By Lemma [13, Lemma 1.14], $\operatorname{spt}(T)$ has $\mathcal{H}^{2 k}$-locally finite measure, so King's theorem follows from (1).

Corollary 3.12. Let $X$ be a complex manifold. If $T \in R R_{k, k}^{l o c}(X)$ is a d-closed, positive real rectifiable current and $n(T, a)$ is either 0 or larger than $b$, where $b>0$ for all $a \in X$, then $T$ is a holomorphic chain with real coefficients.

Proof. Because the result is local, we may consider $X=U$ for some open subset $U \subset \mathbb{C}^{n}$ and $\mathbb{M}(T)<\infty$. Let $N=\{a \in U: n(T, a)>0\}$. Then

$$
T(\varphi)=\int_{N}\langle\varphi(x), \vec{T}(x)\rangle n(T, x) d \mathcal{H}^{2 k}(x)
$$

Hence

$$
b \mathcal{H}^{2 k}(N) \leq \mathbb{M}(T)<\infty .
$$

Therefore by Theorem 3.10, $T$ is a holomorphic chain with real coefficients. 


\section{Applications}

Let $X$ be a compact complex manifold. It follows from a well known result of Federer and Fleming $[5,7]$ that the homology $H_{\star}(\mathscr{D} !(X ; \mathbb{R}))$ of the chain complex $(\mathscr{D} !(X ; \mathbb{R}), d)$ is isomorphic to the singular homology $H_{\star}(X ; \mathbb{R})$ with real coefficients. In the following, $H_{\star}(X ; \mathbb{R})$ denotes the homology of the chain complex $(\mathscr{D}$ ! $(X ; \mathbb{R}), d)$.

Proposition 4.1. Let $X$ be a complex projective manifold of complex dimension $n$ and $e \in A^{n-k, n-k}(X)$ is a $d$-closed form. If e considered as a current has the following property:

$$
e=R+d d^{c} b
$$

where $R$ is a current such that the $(k, k)$-part $R_{k, k}$ of $R$ is positive and $R_{k, k}$ has $\mathcal{H}^{2 k}$-locally finite support, then $e$ is homologous to some algebraic cycles with real coefficients.

Proof. Since $R_{k, k}$ is positive, it is normal. The support of $R_{k, k}$ is of $\mathcal{H}^{2 k}$-locally finite and $d R_{k, k}=0$, by Theorem 2.12, $R_{k, k} \in R R_{k, k}(X)$, and by Theorem 3.10 and Chow's Theorem, it is an algebraic cycle with real coefficients. The result follows from the fact that $e=R_{k, k}+d d^{c} b_{k+1, k+1}$ where $b_{k+1, k+1}$ is $(k+1, k+1)$-part of $b$.

Theorem 4.2. Let $X$ be a complex projective manifold of dimension $n$ and $e \in A^{n-k, n-k}(X)$. If e as a $k$-current is homologous to a Lipschitz $2 k$-chain $P$ with rational coefficients which is $d$-and $d^{c}$-closed and the $(k, k)$-part of $P$ is positive, then e is homologous to an algebraic cycle with rational coefficients.

Proof. By assumption we have

$$
e=P+d a
$$

for some $a \in \mathscr{D}_{2(k+1)}^{\prime}(X)$. Since $d^{c} d a=0$, by the $d d^{c}$-lemma, there is $b \in \mathscr{D}_{2(k+1)}^{\prime}(X)$ such that

$$
d a=d d^{c} b
$$

and hence

$$
e=P+d d^{c} b
$$

Since $\mathcal{H}^{2 k}(\operatorname{spt}(P))<\infty$, and by the assumption, its $(k, k)$-part is positive, hence $P$ fulfills the hypothesis of Proposition 4.1 and therefore $e$ is homologous to an algebraic cycle with real coefficients.

Note that by the assumption on $e,[e] \in H_{2 k}(X ; \mathbb{Q})$. Let

$$
C_{k}(X ; \mathbb{Q}) \subset H_{2 k}(X ; \mathbb{Q}), \quad C_{k}(X ; \mathbb{R}) \subset H_{2 k}(X ; \mathbb{R})
$$

be the subspaces generated by algebraic cycles with rational and real coefficients respectively. Since we may find a basis for $C_{k}(X ; \mathbb{R})$ from algebraic cycles with integral coefficients, these algebraic cycles also form a basis for $C_{k}(X ; \mathbb{Q})$, then we have the equality

$$
C_{k}(X ; \mathbb{Q})=H_{2 k}(X ; \mathbb{Q}) \cap C_{k}(X ; \mathbb{R})
$$

Applying the above observation to our case, we have $[e] \in H_{2 k}(X ; \mathbb{Q}) \cap C_{k}(X ; \mathbb{R})$, and hence in $C_{k}(X ; \mathbb{Q})$.

In the forthcoming paper [22], we formulate a version of the Hodge conjecture in Bott-Chern cohomology and combine results from this paper and results from [21] to prove it.

Conflict of interest: Authors state no conflict of interest.

\section{References}

[1] H. Alexander, Holomorphic chains and the support hypothesis conjecture, JAMS, 10, No. 1(1997), 123-138. 
[2] J.-P. Demailly, Complex analytic and differential geometry, lecture notes on the webpage of the author.

[3] T.-C. Dinh and M.G. Lawrence, Polynomial hulls and positive currents, Ann. Fac. Sci. Toulouse Math., XII(2003), 317-334.

[4] H. Federer, Some theorems on integral currents, Trans. of AMS, Vol. 117(1965), 43-67.

[5] H. Federer, Geometric measure theory, Springer-Verlag, New York, 1969.

[6] H. Federer, Real flat chains, cochains and variational problem, Vol. 24, No. 4(1974), 351-407.

[7] H. Federer, W. H. Fleming, Normal and integral currents, Ann. Math., 72, No. 3(1960), 458-520.

[8] E. Friedlander and H.B. Lawson, A theory of algebraic cocycles, Annals of Math., 136(1992), 361-428.

[9] R. Harvey, Holomorphic chains and their boundaries, Proc. Sym. Pure Math., 30 part 1 (1977), 309-392.

[10] R. Harvey and H.B. Lawson, On the boundaries of complex analytic varieties, Ann. of Math., 102(1975), 233-290.

[11] R. Harvey and H.B. Lawson, A introduction to potential theory in calibrated geometry, Amer. J. Math. 131, no. 4 (2009), $893-$ 944.

[12] R. Harvey and H.B. Lawson, Duality of positive currents and plurisubharmonic functions in calibrated geometry, Amer. J. Math. 131, no. 5 (2009), 1211-1240.

[13] R. Harvey and B. Shiffman, A characterization of holomorphic chains, Ann. of Math., 99, No. 3, (1974), 553-587.

[14] J. R. King, The currents defined by analytic varieties, Acta Math. 127(1971), 185-220.

[15] S. G. Krantz and H. R. Parks, Geometric integration theory, Birkhauser, 2008.

[16] H. B. Lawson, The stable homology of a flat torus, Math. Scand., 36(1975), 49-73.

[17] H.B. Lawson, Algebraic cycles and homotopy theory, Annals of Math., 129(1989), 253-291.

[18] L. Simon, Lectures on Geometric Measure Theory, Australian National University Centre for Mathematical Analysis, Canberra, 1983.

[19] Yum-Tong Siu, Analyticity of sets associated to Lelong numbers and the extension of closed positive currents, Invent. Math., 27(1974), 1-2, 53-156.

[20] G. Stolzenberg, Volumes, limits, and extensions of analytic varieties, Lecture Notes in Math., Vol. 19, 1966.

[21] Jyh-Haur Teh, Chin-Jui Yang, A characterization of real holomorphic chains and applications in the study of algebraic cycles, Complex Manifolds, 7(2020), 93-105.

[22] Jyh-Haur Teh, Chin-Jui Yang, Bott-Chern homology, Bott-Chern differential cohomology and the Hodge conjecture, to appear in the Proceedings of the 8th ICCM. 